\title{
Sobre la sociedad civil como fuente de pluralismo: interrogantes previos a una afirmación.
}

\section{About civil society as source of pluralism: questions before an affirmation.}

\author{
MAXIMILIANO REYES LOBOS \\ Universitat de València
}

Recibido: 21/09/2018Ａceptado: 4/03/2018

\section{RESUMEN}

Frente a los riesgos del monopolio de significados sobre la vida en común, la sociedad civil puede actuar como fuente de reconocimiento y desarrollo de identidades, pero afirmarlo requiere que previamente se establezcan nuevos puntos de partida para el análisis sobre su significado. Apoyados en esta premisa, proponemos un cuestionamiento a la apropiación teórica y práctica que desde la dimensión política se ha hecho y establecemos la redefinición de lo cívico como el sustento para un tipo de pluralismo basado en la capacidad para expresar y conocer por medio de la razón.

\section{PALABRAS CLAVE}

SOCIEDAD CIVIL, DOMINIO, PLURALISMO, EXPRESIVIDAD, RAZÓN.

\begin{abstract}
Faced with the risks of the monopoly of meaning over life in common, civil society can act as a source of recognition and development of identities, but to affirm it, is necessary to establish new starting points for the analysis of its meaning. Based on this premise, we propose a questioning of the theoretical and practical appropriation that from the political dimension has been made and we establish the redefinition of the civic as the sustenance for a type of pluralism based on the ability to express and know through reason.
\end{abstract}




\section{KEYWORDS}

CIVIL SOCIETY, DOMINION, PLURALISM, EXPRESSIVENESS, REASON.

\section{SOBRE LO NORMATIVO Y EL PREDOMINIO}

DeSDE LA POLITIKE KOINONÍA ARISTOTÉLICA, hasta la propuesta de una sociedad civil protagonista de la definición social de la sociedad (Keane, 2003; Jeffrey, Staeheli y Marshall, 2018), el carácter asociativo de las relaciones humanas ha servido de fundamento para la elaboración de teorías filosófico-políticas y la defensa de puntos de vista respecto a los motivos por los cuales el ser humano ha de proceder de determinada manera en ciertas circunstancias. Algunos de estos puntos de vista han centrado su atención en la colectividad como propósito orientador de la conducta pública y privada, pudiendo incluso instrumentalizar al individuo, mientras que otros han abogado por realzar las virtudes del sujeto en particular como sustento de la trama de relaciones, aunque no por ello, han evitado los riesgos de reducir lo social a una suma de actuaciones personales (Gracia, 2010).

Sea cual fuere el caso, es posible apreciar en cada uno, una la tendencia a fijar normativamente modos de asociación que respondan a los principios que la teoría indica. Gracias a ello, hoy puede hablarse, por ejemplo, de la imposibilidad de que el hombre pueda situarse en el mundo sin necesidad de contrapartes con quienes establecer lazos, lo cual, más allá de la tradición que la exponga, revela la importancia de contar con una conceptualización clara sobre la facultad de asociación humana.

Por ello, si hay un aspecto que es fundamental y merece ser destacado, es que la búsqueda de identidad de la sociedad civil nace con la búsqueda de la identidad de los individuos y colectivos que la conforman, lo cual nos orienta a reflexionar sobre las nociones de igualdad y diferencia que forman parte de la definición de sociedad civil.

En su libro Las esferas de la justicia. Una defensa del pluralismo y la igualdad, Michael Walzer (1997) expuso que la lucha por la igualdad no finalizaría con el logro de una sociedad igualitaria. Más bien, el escenario a alcanzar en este caso sería uno donde las disputas disminuirán y la lucha se suavizará dada la habituación de mujeres y hombres a vivir autónomamente y apreciando que una sociedad podrá ser justa en la medida que se reconozca la diferencia en los resultados obtenidos por diversos sujetos en contextos distintos.

No obstante, dado el caso que un grupo de mujeres y hombres lograsen monopolizar los medios para ciertos resultados y relativizar las maneras en que otros hombres y mujeres ejercen su autonomía en sus contextos, ¿pueden ser, a su vez, los bienes monopolizados y reinterpretados desde un significado 
extrínseco a causa de su predominio por sobre otros bienes? Nos referimos a bienes tales como la tierra, el capital, el poder político o el carisma; aspectos que en un escenario como el planteado por Walzer, se superponen e intercalan en diferentes esferas de actuación, de acuerdo con los significados que los propios sujetos integrantes de tales esferas les otorgan y no según los que son establecidos desde una posición de dominio.

Una situación tal, en la que la igualdad entre las personas se traduzca en la equivalente libertad para ser distintos, puede ser considerada como el propósito que persigue una sociedad con aspiraciones de justicia (Siurana, 2009). Sin embargo, el pronóstico sociopolítico nos muestra una realidad en la que los bienes son acaparados por un número reducido de sujetos que por medio de su acción, determinan la de la mayoría. Por ello, y sin tener la intención de caracterizar la situación de dominio en la amplitud y variedad de bienes existentes, nos remitiremos en este estudio a aquellos elementos que consideramos fundamentales para las definiciones que la ciudadanía realiza, en tanto que sociedad civil.

\section{EXTERNALIDAD VS PRAXIS}

Dependiendo de la concepción de democracia que predomine en un determinado sistema sociopolítico, el perfil de sociedad civil que a través de él se configura, lo podrá hacer brindando espacios para la participación de todos los ciudadanos - a modo aristotélico-comunitarista, podríamos decir-o delimitando a priori el acceso a espacios donde desarrollar una vida pública y compartida. De darse el primer caso, hablar de ciudadanía y civilidad significaría hablar de comunidad, empero, creemos que es más factible que nuestra sociedad occidental se ordene según el significado extrínseco que se le otorga a la esfera política, sobre todo en términos de definiciones provistas desde regímenes y desde élites, sean éstas civiles o políticas (Delamaza, 2013), o que aún en el caso de una postura comunitarista, aquella sea definida desde el poder monopolizador del aparato estatal (Barber 2000).

Con ello, puede plantearse que con independencia de la tradición desde las que se examine el asunto, sea en términos de liberalismo, republicanismo o comunitarismo, la determinación del obrar cívico se establece desde el predominio de un modelo operativo y el lineamiento paradigmático de una ideología política y no desde el modelo de acción que es propio de la praxis ciudadana. En este sentido, cuando nos referimos a que la política, entendida como un bien, es monopolizada, aludimos a que desde su predominio sobre otros bienes, como lo pueden ser el carisma, la intersubjetividad o la díada mano de obra-tierra, la configuración de la sociedad civil se realiza desde la externalidad. Es decir, desde pautas ajenas a ella misma y con una distinguible presión que quienes han monopolizado el bien político, ejercen sobre la sociedad civil, regulando 
las formas y normas por las cuales la ciudadanía asume saberes y prácticas ya institucionalizadas. En este caso, el riesgo de monopolio y predominio del bien político es la singularización de la sociedad civil y la aceptación de que el ciudadano es inherentemente débil, por lo que su inclusión en la colectividad se fundamenta en la afirmación de ideales externos a su capacidad de conceptualización (Durkheim, 2006; 2018).

Si por una parte, desde una postura comunitarista la libertad se entiende como participación, desde el republicanismo es caracterizada como pertenencia y desde el liberalismo se concreta en la no-interferencia, por otra, estas tres perspectivas comparten el hecho de que la definición de la sociedad civil es provista por la tradición en particular -desde lo singular-y no desde la dinámica interna y relativa a la ciudadanía que la conforma -desde lo plural (Cfr. Walzer, 1998; Reyes, 2019). Ello puede derivar tanto en que el análisis de la sociedad civil restringe sus fines a un ideal únicamente deliberativo, con lo cual se invisibilizan los elementos más profundos de agencia cívica e interacción humana (Dahlgren, 2006), como en una ficticia propuesta de globalizacion ciudadana que responde a intereses neoliberales (Munck, 2006)

Ahora bien, en favor de una dinámica ciudadana, podemos notar que los hombres, como sujetos capaces de comprender que su vida colaborativa implica respetar los ideales de vida buena de sus conciudadanos, lo hacen a sabiendas de que por muy distintos que sean estos ideales, todos son válidos y merecen consideración ateniéndose a conformar unos ideales mínimos compartidos (Cortina, 1994). Sin embargo, asumir esto como parte de la argumentación requiere indagar en sí, efectivamente, es la capacidad asociativa del ser humano la que podrá detener el avance de un predominio que desconozca las virtudes de la multiplicidad de formas de ser y de estar en el mundo, con lo cual se abogaría por una red de asociaciones que sirva de escenario para la expresión de identidades y la construcción de lazos sociales fuertes y complementarios.

Cierto es que «cada sociedad es particular en su conformación y en las pautas de su desarrollo» (Delamaza, 2013, p. 7), pero precisamos aún recabar en las implicancias de una sociedad civil que sea garante de ello, a fin de no desconocer los conflictos y limitaciones que esta multiplicidad, que nosotros conceptualizamos como pluralismo, puede exhibir.

En coherencia con ello, y sin detenernos en las definiciones que nos aporta la discusión teórica, comprendemos que en la práctica, la sociedad civil contiene y se configura a partir de una diversidad de escenarios, sujetos, instituciones y sistemas, que se diferencian unos de otros de acuerdo a su grado de formalidad, a las relaciones de jerarquía y autonomía, y fundamentalmente, según la noción y uso del poder (Adrian, 2009; Flyvbjerg, 2012; Anheier, 2014). 


\section{TRAS EL PLURALISMO}

Si una sociedad opta por definirse como pluralista, asumimos que no es solamente porque ha superado una etapa caracterizada por la existencia de un código moral único que norma la forja de identidad y las interacciones entre los individuos, sino que más profundamente, lo es porque el reconocimiento a la autenticidad y la existencia de valores morales que orientan su respeto, ha permitido que lo haga. Sería, por tanto, el pluralismo el que protagoniza la estructuración de las interacciones libres entre individuos, definiéndose por la voluntad común ciudadana y no por la simultaneidad de opiniones sobre asuntos determinados extrínsecamente y ante los cuales los individuos están mandatados para responder cuando es necesario (Cortina, 1994). Pero a pesar de esta posiblidad, alcanzar un nuevo escenario con estas condiciones supone tanto el conocimiento de las diversas formar de comprender la moral como sustantivo y como adjetivo (Cortina y Martínez, 2015), como la conflictualidad funcional y disfuncional propia de la naturaleza humana (Rabinow, 1984; Fernández-Ríos, 1999). ${ }^{1}$

Sólo tomando como referencia la relación entre Ética y Política podemos desde ya plantear interrogantes que conjugan en sus posibles respuestas, la complejidad de la naturaleza del ser humano en su pertenencia social. A este respecto, en unos casos, el predominio de lo político implica que la política cuenta con sus propias leyes separadas de la moral, por lo cual el obrar político no está sujeto a juicio ético (Maquiavelo, 1988; Laporta, 1990). En otros, las acciones privadas de los hombres sólo les atañen moralmente a ellos, lo cual conduce a concluir que existiría una moralidad privada y una moralidad pública, reduciéndose cualquier definición de la sociedad civil a si ella puede intervenir en las acciones privadas de los hombres y mujeres que no causan daño a otros. Según esto, «la individualidad debe gobernar aquella parte de la vida que interesa principalmente al individuo, y la sociedad esa otra parte que interesa principalmente a la sociedad» (Mill, 2016, p. 91). Pero si esto ha de ser así, ¿cómo se pueden reconciliar los ideales de unos y otros individuos al establecer una política de gobierno en particular? (George, 2005).

En consecuencia, preguntarnos si la sociedad civil puede ser fuente de pluralismo, implica cuestionar primeramente las posibilidades de un mínimo moral e indagar en la esencia del reconocimiento, pues entendemos que es a partir de ello, que más tarde se podrán establecer nuevas líneas de investigación sobre las dimensiones local y global de la sociedad civil o respecto a la

1 Es decir, la tendencia inherente del ser humano a relacionarse en torno al establecimiento de conflictos, los cuales en unos casos pueden servir para cumplir los objetivos de los involucrados y beneficiarlos (funcional), mientras que en otros, son perjudiciales para unos y otros, dado que no sirven para, o no se corresponden con los fines que se desean. 
adscripción a una tradición teórica en específico. Por lo pronto, corcordamos en que si la sociedad civil aspira a posicionarse como un núcleo ético de la sociedad y a actuar como garante de convivencia sociopolítica, sin derivar en una aglomeración de perspectivas carentes de un criterio que la determine, entonces las principales teorías que la han tratado deberían someterse a revisión, al tiempo que se la concibe como un campo de investigación fructífero e intelectualmente atractivo (Feenstra, 2010; Anheier, 2014)

No es nuestra intención realizar en estas líneas tal revisión; sólo consideramos pertinente trazar ciertos condicionantes que al ser analizados desde una tradición y desde la mopolización de la política, se interpretan como obstaculizadores del pluralismo en la sociedad civil, pero que al ser tratados desde la esencia del civismo en sí -es decir, desde la tendencia a compartir ciertas pautas de convivencia social- son comprendidos como facilitadores de una nueva dinámica social basada en el reconocimiento a la multiplicidad de expresiones y de saberes (Reverter-Bañón, 2006; Gready y Robins, 2017).

Por tanto, si es la sociedad civil el ámbito de interacciones por medio del cual se rigen las pautas de la organización social, entonces ¿cuáles son las cualidades que este ámbito posee y que patrocinan a los individuos en la creación y recreación de relaciones sociales?

Desde una posición voluntarista, podríamos afirmar que el pluralismo es el lugar común para la existencia de mínimos morales que sean compartidos aun cuando no se tenga la misma concepción sobre lo que significa una vida buena. Pero de ser así, ¿cómo conjugar las distintas concepciones de vida y de felicidad que pueden nacer en el seno de la sociedad civil, con una única noción sobre los mínimos de, por ejemplo, justicia? Una respuesta generalizada, apunta a que pluralismo es mostrar libremente los ideales propios y no imponerlos. Es dialogar sobre ellos y no discriminarlos. Pero entenderlo de este modo obliga a plantear el interrogante respecto a lo que se entiende por diálogo y en específico, a los significados que cada individuo posee sobre lo que se quiere acordar. Con tal apreciación, surgen más inconvenientes que certezas respecto a la cabal espontaneidad del individuo para establecer diálogo. Pareciera más bien, que la formación de la sociedad civil se desarrolla como un proceso político que, buscando su cimiento en la comprensión que los sujetos poseen sobre cómo debe ser la política en términos ideales o deseables, lo hace por el contrario, desde esquemas generales y con escenarios de discusión donde prevalence la representatividad (Schudon, 2012).

Pero «el individuo tiene necesidad, para ser él mismo, de ser reconocido» (Taylor, 1996, p. 13), por lo que hablar de reconocimiento conlleva necesariamente hablar sobre su cariz constructivo de identidad y sobre la reciprocidad que le es inherente como una cualidad del ser humano que se manifiesta tanto a nivel individual como en la interacción con otros. Y el surgimiento del reco- 
nocimiento como una respuesta a la necesidad de expresión de cada modo de ser humano, ocurre como un proceso de participación del individuo y del otro en una búsqueda de un ideal de autenticidad y de fidelidad hacia uno mismo en un escenario de horizontes variables.

Desde este punto de vista, en la medida que un individuo forja su yo en la expresión de la propia autenticidad y en la interacción con otros, el concepto de identidad individual y colectiva tendrá un común denominador que permite relevar la manera en que cada ser humano participa en la (re)definición del horizonte moral en el que se sitúa. De este modo, si «la esfera del reconocimiento se extiende en principio al infinito en el caso de los pueblos» (Taylor, 1996, p.18), la importancia de que individuos se reconozcan mutuamente como sujetos particulares y a la vez como miembros de un colectivo que posee su propia identidad, radicaría en que se le otorgue a la sociedad civil la responsabilidad de resguardar la esfera del reconocimiento, esto dado que sus fronteras son determinadas únicamente por el alcance y profundidad de los conflictos y acuerdos que en ella surgen (García Marzá, 2008).

No obstante, la identidad individual que se construye en estos términos no se traduce necesariamente en que el reconocimiento es recíproco ni en que esta identidad encuentra su equivalencia en términos colectivos, ya que aún es posible que en la interacción con otros, un individuo pueda ser juzgado con el criterio de cumplimiento o incumplimiento de determinadas pautas, o que lisa y llanamente no sea juzgado y por tanto, no sea reconocido. Este fenómeno de no-reconocimiento no sólo se interpreta como una falta de respeto por la existencia de un individuo distinto, sino que causa en éste un trauma identitario; un profundo odio a sí mismo (Taylor, 2009).

Es por tales motivos que esta concepción constructiva del reconocimiento no siempre se ha planteado como una cualidad de la organización social con miras al libre acuerdo de los individuos. Si bien, convenimos en que la expresión de la autenticidad y el sentido de pertenencia que experimenta un individuo al ser reconocido por los otros, es garante de la cohesión grupal, comprendemos a su vez, que el reconocimiento puede aún estar limitado por el cumplimiento de ciertos criterios de honor y por la pertenencia a determinados rangos sociales, lo cual mediado por el dominio del componente político en la definición de la sociedad civil, puede concluir en la instauración de fenómenos de asimilación, segregación y exclusión social (Cfr. Berry, 1997).

Dicho esto, ¿es el pluralismo «la condición indispensable de la libertad en una sociedad civil fuertemente democrática»? (Barber, 2000, p. 45). Tratarlo así, se traduciría en que para que la sociedad civil se configure como una red de asociaciones vigorosa, necesita que primeramente se potencie una ética que sea compartida por todos los miembros de la sociedad, ya que la inexistencia de ciertos mínimos morales compartidos no permite a los individuos 
convertir su participación y el reconocimiento de sí mismos y de los otros, en el ejercicio de una ciudadanía efectiva. Es por ello que se precisa apelar a una transformación de la sociedad, de modo que el paso de un monismo moral a un pluralismo moral involucre asunciones de responsabilidad en dos sentidos: por una parte, adjetivar la responsabilidad en términos éticos para diferenciar nuestra conducta de la conducta animal, reconociendo con ello la importancia de distintos códigos morales que siendo relativos a cada una de las diversas interacciones que estructuran la sociedad civil, se complementan en el diálogo y en la afirmación de la identidad colectiva (Cortina, 1994; Cortina y Martínez, 2015). Por otra, asumir que con independencia de la interpretación que se haga del componente político de las ideologías - de las cuales se desprenden las tradiciones políticas (Conill, 1998)-, el obrar de la sociedad civil se interpreta no sólo en términos normativos, sino que también lo es en términos de significados, prácticas, costumbres e identidades. En suma, en términos culturales (Dahlgren, 2006).

Es en este sentido que adquiere relevancia un pluralismo basado en que «el significado de algo sólo se adquiere a través del contraste con otras cosas» (Gracia, 2010, p. 202), por lo que junto con proveer los espacios que la convivencia necesita para que se compartan los mínimos morales, es propicio establecer otro tipo de espacio en el cual el vínculo comunicativo cuente con un horizonte de significado compartido. Es necesario que los individuos puedan desarrollar un entendimiento mutuo basado no en su capacidad de pensamiento como cualidad restringida a cada sujeto particular, sino como una capacidad que se modela en la interacción y confrontación misma con los demás.

En este escenario, la controversia es aceptada, porque la sociedad civil como fuente pluralismo no se define únicamente según una concepción de poder $\mathrm{y}$ control en términos foucaultianos, ni tampoco lo es en perspectiva habermasiana, como un exclusivo ámbito de interacciones de carácter voluntario. Y esto dado que, si bien por una parte, a lo que se aspira con una postura consensual de sociedad civil es a una definición basada en la reciprocidad entre sus miembros y en el respeto a la diversidad de fuentes de asociación y organización correspondientes a cada esfera, la propuesta voluntarista tiende a alejarse de lo real y lo contextual al omitir respuestas a interrogantes sobre, por ejemplo, dónde está el poder o quien lo ejerce (Feenstra, 2008; Flyvbjerg, 2012). Por ello, aun cuando aceptemos que el horizonte moral que determina la relación consensual puede ajustarse a la variedad de intereses, sean estos particulares, comunes o universales (García Marzá, 2008), opinamos que la aspiración de esta postura no satisface completamente los requerimientos de una definición de lo moral, siendo la moral por sí misma una noción cuya polisemia impide determinar el curso de la sociedad civil desde una única perspectiva, por muy garante de consenso que parezca. 
Pero lo señalado no debe entenderse como una total argumentación en favor de posturas teóricas disímiles, ya que por otra parte, si bien las perspectivas basadas en el carácter del poder y del dominio de influencia foucaultiana, o las que caracterizan a la sociedad civil en términos histórico-contextuales son acertadas al centrar su atención en los procesos por los cuales se configuran las asociaciones ciudadanas (Ehrenberg, 1999), pueden al mismo tiempo, promover el predominio de ciertos criterios de pertenencia al definir a la sociedad civil según qué actores forman parte de ella y quienes quedan excluídos.

Más ampliamente, argumentamos que como fuente de pluralismo, la sociedad civil no debería definirse por estas posturas, sino que por el contrario, ha de contenerlas y configurarse como el punto neurálgico desde el cual se pueden determinar las relaciones en términos sociales y políticos (Jeffrey, Staeheli y Marshall, 2018). Esta reflexión abre la posibilidad de que la sociedad civil pueda ser entendida como el lugar común en el cual la relación que surje es una armonía de contrarios que genera un constante movimiento de contraposición y conflicto; una relación que nunca genera saberes absolutos, ni es en sí mismo un movimiento concluso; una oposición que moviliza y dinamiza, más nunca paraliza, ya que hacerlo significaría alcanzar un no-movimiento y por ende, una conclusión (Editorial Gredos, 1981; Cfr. Adorno, 1984).

Lo anterior presupone que el horizonte donde el significado es compartido no es reductible a la experiencia individual de cada uno de los miembros de las distintas asociaciones de la sociedad civil, sino que como un todo unificado, se establece como el resultado de las interacciones como un equivalente a la intersubjetividad. Por tanto, la garantía de convivencia que hace posible que las personas puedan compartir unos mínimos morales se encuentra en la indivisibilidad del horizonte de significado en interpretaciones individualistas. Así mismo, el respeto a los ideales de otro individuo por muy diferentes que sean de los propios, no se funda en los mínimos morales a los que se aspira -aun cuando el respeto en sí mismo sea un mínimo moral-, sino que lo hace antes que todo en la mutua identificación en el uso de un lenguaje que se construye en la interacción y cuyo telos está en su práctica misma, no es su instrumentalización (Gracia, 2011).

Lo anterior respalda que dado que los mínimos morales se definen y comparten en la interacción entre individuos, es necesario a su vez que estos cuenten con una capacidad expresiva que sirva de trasfondo para el respeto por los ideales que se acuerdan. Es ir más allá de la diferencia entre fenómenos morales e interpretaciones morales de los fenómenos nietzscheana. Más bien, involucra la búsqueda de un cariz ético orientador en cada ámbito de quehacer humano, los cuales aún estando delimitados por las distintas asociaciones voluntarias a las que el hombre se adhiere, se unifican en el significado que se comparte. 


\section{EXPRESIVIDAD Y SABER}

Ahora bien, ¿cómo se construye este trasfondo de significado compartido en la sociedad civil? Si retomamos la crítica inicial al predominio del componente político representado en las posturas liberales, republicanas y comunitaristas, podemos analizar en la misma línea, que es a contar del siglo XVIII que el surgimiento de la noción de autenticidad se posiciona como el momento a partir del cual la naturaleza humana se redefine en un sentido moral, por cuanto a que a partir de esta centuria el ser humano es concebido como dotado de un sentido intuitivo acerca de aquello que es bueno y aquello que es malo. Es a partir de este momento, que pareciera como si el ser humano tuviese una voz interior que le dicta lo que ha de hacer y lo que no en función de las nociones sobre lo bueno y lo malo, por lo que la autenticidad se plantea como la cualidad de atender a esta voz, desarrollar ciertos principios orientadores y serles fiel. Es entonces esta fidelidad a lo interior, a la fuente, a lo propio en términos morales, lo que define al hombre moderno.

La referencia a Rousseau que se hace es clave para entender este desarrollo moral, en tanto que importa atender a la voz de la naturaleza que existe al interior de cada individuo. Pero creemos que es más importante la adherencia a la idea herderiana sobre el modo original que cada ser humano exhibe y que determina la irrepetible medida que tiene cada individuo (Taylor, 2009). Esta idea configura la identidad como un modo de ser que es propio a cada persona y que por lo mismo, en la expresión de su originalidad cuenta con una voz única que ha de manifestarse.

No obstante, si el ser fiel a uno mismo se traduce en ser fiel a la voz moral que reside dentro de cada uno y el ideal de autenticidad es el modo de ser único y original configurado internamente, ¿de qué manera el ser humano se relaciona con otros, reconociéndolos y desarrollando una identidad compartida? Es preciso tomar en cuenta un rasgo de la condición humana que participa en la fidelidad a la voz moral pero que no se percibe en su cabal relevancia cuando se incorpora la participación de otro sujeto en la forja de la identidad, tanto individual como compartida. Este rasgo es el carácter dialógico de la vida humana que se evidencia en el acto de atender a la voz moral interior, pero que en la relación con otros individuos require de mayor atención.

Lo que se esperaría es que la expresión de la autenticidad encuentre su razón de ser en el intercambio con otros y que sea mediante la relación dialógica que la identidad se construya y sea reconocida por los demás.

Aludiendo nuevamente a la crítica al predominio de lo político, sería esta concepción expresivista del lenguaje la que diferencia a la sociedad civil del Estado, por cuanto a que éste exhibe un ordenamiento estamental que no requiere de la identidad ni del reconocimiento para definirse. Más bien, en él 
el lenguaje se torna en una actividad productiva, mientras que en la sociedad civil «el lenguaje no se concibe como el ensamble de palabras, sino como la capacidad para expresar la conciencia reflexiva implícita en el uso de las palabras para decir algo» (Gracia, 2010, pp. 201-202).

Sin embargo, abogar únicamente por esta cualidad de la sociedad civil puede conducir al lector a la inevitable cuestión respecto a si en ausencia o en reemplazo de sociedad civil, es el gobierno de las leyes capaz de dar garantía de los derechos individuales y si el acto de restringir la intervención del Estado en favor de la libertad del individuo favorece una identificación de los individuos entre sí y de todos como un solo colectivo (Gracia, 2011). Frente a estas cuestiones, profundizar en la capacidad dialógica implica preguntarse si el comportamiento de los individuos se rige por principios jurisdiccionales o por principios morales. De ocurrir lo primero, la organización de la sociedad como ámbito de interacciones humanas estaría mediada por la primacía del derecho sobre la civilidad, mientras que de suceder lo segundo, la sociedad civil y el reconocimiento que en ella fructifica, se regirían por la construcción del carácter, por la participación en los asuntos de interés público y por el significado que se comparte sobre ellos.

Si bien, la razón práctica puede orientar el proceder de la sociedad civil, facultándola para elegir aquello que pueda convertirse en ley universal, también es cierto que ella parte del supuesto de que en dicha comunidad el diálogo se da entre sujetos morales, racionales y con perfecta capacidad deliberativa. Es decir, la prioridad del razonamiento cívico sobre la política obviaría la imperfección y la cotidianidad inherente al actuar del individuo en el tratamiento de lo justo y lo bueno. Si a este escenario se le otorga la relevancia que se merece, es clave tener en cuenta que la política provee un necesario poder para ordenar y asegurar la paz y el derecho (Del Águila, 2000). Es su prioridad, entonces, un rescate a la discordancia que define las relaciones democráticas y una muestra que la duplicidad entre la capacidad de actuar y la diversidad de nociones de lo justo y lo bueno, forman parte del ordenamiento político. No es de extrañar que con ello, el juicio que la ciudadanía realiza libremente sobre la pertinencia de una decisión tomada desde el aparato estatal de forma a una razón cívica.

En otras palabras, la racionalidad que se pueda observar en la sociedad civil sería una racionalidad que redefine la identidad política que se ha configurado primeramente en lo estatal, teniendo como campo de aplicación la interpretación, la deliberación y la proyección (Del Águila, 1995). Y sería el reconocimiento a esta discordia desde donde se deriva una razón a posteriori que se configura a través de la experiencia que surge en la sociedad civil. Ello nos conduce a asumir que en la investigación sobre la sociedad civil se debe relevar el componente empírico y no sólo la teorización. $\mathrm{Y}$ es posible que sea esta razón, el principal argumento en favor del interés de las personas por 
formar pactos por el bien común (Calhoun, 1994; Power, Muddiman, Moles y Taylor, 2018). Comprendemos que la sociedad civil como fuente de pluralismo no concibe la posibilidad de una cabeza no sometida a leyes, ya que aun cuando ella no pueda decretarlas, sí es quien por medio de su saber y su expresión, indica a un cuerpo administrativo la regulación jurídica de la sociedad. Es de este modo, como lo normativo se estructuraría desde la influencia de lo cívico sobre lo jurídico; desde lo práctico hacia lo teórico; desde lo contextual e histórico hacia lo generalizable, concretándose en el conjunto de saberes (dinámicos, contextualmente originados, generalizables, no concluidos, ni absolutos) que son la materia prima con la cual la sociedad civil genera el movimiento político por el cual ejerce influencia en el aparato estatal. Pero no por ello, lo opuesto no sucede. Por el contrario, en las bases de un pluralismo cívico encontramos la superación de la escisión de la sociedad según grupos e intereses, aceptándose los contrastes dada la unidad superior que se logra por medio del saber y la expresión.

Es en consideración a lo dicho que desde una perspectiva pluralista, la libertad puede ser entendida como expresividad y como saber. No nos referimos a la libertad de expresión y a la acumulación de información, sino más bien, a un capacidad expresivista; a la libertad como la capacidad para expresar las determinadas y específicas maneras de ser y de concebir el yo y sus relaciones, junto con una disposición a conocer por medio de la razón (Taylor, 2014). La aspiración pluralista de la sociedad civil sería entonces, una aspiración de libertad para desarrollar y exponer una capacidad íntegramente expresivista y radicalmente racional. Una libertad que ha de ser a su vez reconocida, lo que sitúa al reconocimiento como estandarte del pluralismo. De este modo, los efectos del no-reconocimiento que se observan en la construcción de un mundo moral que no considera a los individuos que conforman la sociedad, son símbolo del riesgo que se puede correr al aspirar a una sociedad igualitarista. A fin de cuentas, la moral cívica propia de una sociedad civil la hacen las personas comunes y corrientes, por lo que el reconocimiento no ha de confundirse con la mera tolerancia generosa de la diferencia en el pensamiento y en la actuación de otros, sino que ha de ser la vía por la cual los individuos expresan su identidad, contribuyen a la construcción de la identidad colectiva y articulan una opinión compartida que vigoriza los lazos sociales (Cortina, 1994; Gracia, 2011).

\section{REFERENCIAS BIBLIOGRÁFICAS}

Adorno, T. W. (1984). Dialéctica negativa. Madrid: Taurus.

Adrian, Á. (2009). Civil Society in Second life. International Review of Law, Computers y Technology, 23(3), 231-235. 
Sobre la sociedad civil como fuente de pluralismo...

Anheier, H. K. (2014). Civil Society Research: Ten years on. Journal of Civil Society, 10(4), 335-339.

Barber, B. R. (2000). Un lugar para todos. Cómo fortalecer la democracia y la sociedad civil. Barcelona: Paidós.

Berry, J. W. (1997). Immigration, Acculturation and Adaptation. Applied Psychology: An International Review, 46(1), 5-68.

Calhoun, E. (1994). Voice in Government: The People. Notre Dame Journal of La, Ethics y Public Policy, 8(2), 427-466.

Conill, J. (1998). Ideologías políticas. En A. Cortina, 10 palabras clave en Filosofía Política (págs. 213-258). Navarra: Verbo Divino.

Cortina, A. (1994). La ética de la sociedad civil. Madrid: Anaya.

Cortina, A., y Martínez, E. (2015). Ética. Madrid: Akal.

Dahlgren, P. (2006). Doing citizenship: The cultural origin of civic agency in the public sphere. European Journal of Cultural Studies, 9(3), 267-286.

Del Águila, R. (1995). Razón de estado y razón cívica. Claves de razón práctica(52), 10-21.

Del Águila, R. (2000). Política, derecho y razón de Estado. Revista Española de Ciencia Política(3), 11-29.

Delamaza, G. (2013). De la elite civil a la elite política. Reproducción del poder en contextos de democratización. Polis. Revista Latinoamericana(36), 1-31.

Durkheim, E. (2006). Sociología y Filosofía. Granada: Comares.

Durkheim, E. (2018). Las reglas del método sociológico y otros escritos. Madrid: Alianza Editorial.

Editorial Gredos. (1981). Los filósofos presocráticos I. Madrid: Editorial Gredos.

Ehrenberg, J. (1999). Civil Society: The Critical History of an Idea. New York: New York University Press.

Feenstra, R. A. (2008). Diferentes concepciones de sociedad civil: la problemática de un concepto. Recerca. Revista de pensament i anàlisi(8), 47-66.

Fernández-Ríos, M. (1999). Aspectos positivos y negativos del conflicto. En F. J. Morales, y S. Yubero, El grupo y sus conflictos (págs. 25-46). Cuenca: Universidad de Castilla-La Mancha.

Flyvbjerg, B. (2012). Habermas and Foucault: Thinkers for Civil Society? En S. R. Clegg, y M. Haugaard, Power, Vol. 1: Power and Politics (págs. 117-140). London: Sage.

García Marzá, D. (2008). Sociedad civil: una concepción radical. Recerca, Revista de Pensament i Anàlisi(8), 27-46.

George, F. (2005). Civil liberties vs National security: The enduring tension. Notre Dame Journal of Law, Ethics y Public Policy, 19(1), 219-232.

Gracia, J. (2010). Posibilidad de un individualismo holista. Consideraciones hermenéuticas sobre el individualismo moderno desde la filosofía de Charles 
Taylor. Isegoría. Revista de Filosofía Moral y Política(42), 199-213.

Gracia, J. (2011). Individualismo y sociedad en la filosofía de Charles Taylor. Una aproximación desde el enfoque hermenéutico. Contrastes. Revista Internacional de Filosofía, XVI, 193-210.

Gready, P., y Robins, S. (2017). Rethinking civil society and transitional justice: lessons fron social movements and «new» civil society. The International Journal of Human Rights, 21(7), 956-975.

Jeffrey, A., Staeheli, L., y Marshall, D. J. (2018). Rethinking the spaces of civil society. Political Geography, 67, 111-114.

Keane, J. (2003). Global Civil Society? Cambridge: Cambridge University Press.

Laporta, F. (1990). Ética y política. Algunas claves básicas. Claves de razón práctica. (2), 15-21.

Maquiavelo, N. (1988). El príncipe. Madrid: Tecnos.

Mill, J. S. (2016). Sobre la libertad. Madrid: Verbum.

Munck, R. (2006). Global Civil Society: Royal road or slippery path? Voluntas(17), 325-332.

Power, S., Muddiman, E., Moles, K., y Taylor, C. (2018). Civil society: Bringing the family back in. Journal of Civil Society, 14(3), 193-206.

Rabinow, P. (1984). The Foucault reader. New York: Pantheon Books.

Reyes, M. (2019). Las fuentes de la sociedad civil. Ciudadanía, ética y politica. Seminario de Ething, ética y coaching. Linares: Museo de Arte y Artesanía de Linares.

Reverter-Bañón, S. (2006). Civil society and Gender equiality: A theoretical approach. Civil Society Working Paper(24), 2-32.

Schudon, M. (2012). The public sphere and its problems: Bringing the state (back) in. Notre Dame Journal of Law, Ethics y Public Policy, 8(2), 529-546.

Siurana, J. C. (2009). La sociedad ética. Indicadores para evaluar éticamente una sociedad. Barcelona: Proteus.

Taylor, C. (1996). Identidad y reconocimiento. Revista Internacional de Filosofía Política(7), 10-19.

Taylor, C. (2009). El multiculturalismo y la «política del reconocimiento». México DF: Fondo de Cultura Económica.

Taylor, C. (2014). Hegel y la sociedad moderna. México D.F.: Fondo de Cultura Económica.

Walzer, M. (1997). Las esferas de la justicia. Una defensa del pluralismo y la igualdad. México D.F.: Fondo de Cultura Económica.

Walzer, M. (1998). La idea de la sociedad civil. Una vía de reconstrucción social. En R. del Águila, y F. Vallespí, La democracia en sus textos (págs. 375-394). Madrid: Alianza. 
Maximiliano Reyes Lobos es psicólogo por la Universidad de Talca, Chile.

Realiza el Máster en Ética y Democracia de la Universitat de València, España..

Lineas de investigación:

Sociedad civil, democracia deliberativa.

Dirección electrónica:

max.reyeslobos@gmail.com 
